\title{
Near Fields around Metallic Walls due to a Nearby Dipole Source with Applications to EMC
}

\author{
Ki-Chai Kim ${ }^{\dagger}$, Sung Min Lim* and Jong-Woo Kim**
}

\begin{abstract}
This paper discusses the near field characteristics of a dipole source located near conducting metallic walls from an electromagnetic compatibility (EMC) point of view. An integral equation for a dipole source near a metallic wall is derived and solved by applying Galerkin's method of moments (MoM). The results show that in the regions outside the dipole source, total electric near fields decrease gradually in magnitude with an increasing field point from the dipole source. But in the regions inside the dipole source, total electric near fields decrease rapidly with a dipole position of $h \leq$ $0.3 \lambda$. For a dipole position of $h \geq 0.7 \lambda$, the peaks and nulls of the total near electric field occur periodically in the regions inside the dipole source, and the fluctuation period is almost $0.5 \lambda$. The worst position for a receptor location is along the $z$-axis, and a range of a half-magnitude of the maximum near electric field in the principal $H$-plane is about two times broader than that of the principal $E$-plane. Experimental measurements are also presented to validate the theory.
\end{abstract}

Keywords: Near field, Dipole source, Metallic wall, Electromagnetic compatibility (EMC)

\section{Introduction}

The study of the near fields of radiating objects has been a subject of interest to electromagnetic compatibility (EMC) engineers for many years [1-3]. Many studies have examined various problems associated with near fields around radiating objects, and method of moments (MoM) can be used to accurately compute the near fields of thinwire structures [4-8]. In many cases, the electromagnetic (EM) source and equipment under test (EUT) receptor are located near a metallic wall (see Fig. 1(a)). The coupling of electromagnetic fields between EM source and EUT receptor closely located to a conducting metallic wall is often encountered, and the model used for our study is a dipole source (which might represent a handheld phone antenna) located near an infinite conducting metallic wall. The dipole source radiates electromagnetic fields, and it affects the EUT located near the metallic wall. The near field's behavior will be a very interesting subject in this situation.

The field distribution in the near, as well as far, zone of an EM source near a metallic wall is useful for estimation of electromagnetic interference with any other electronic system or subsystem. From the EMC point of view, the peaks near the metallic wall should be considered even more carefully to prevent malfunction of the EUT receptor. Consequently, a receptor located near the metallic wall

$\dagger$ Corresponding Author: Dept. of Electrical Engineering, Yeungnam Univerity, Korea. (kckim@ynu.ac.kr)

* SL corporation/R\&D Dept. Electronic Test Team, Korea (smlim@sl.re.co)

** Korea Marine Equipment Research Institute (KOMERI), Korea. (kim0307@komeri.re.kr)

Received: June 20, 2016; Accepted: October 25, 2016 should be treated more carefully than the usual case without a metallic wall.

This paper presents the near field characteristics of a dipole source near metallic walls. The variation of the electric field along the normal to the metallic wall is studied in detail as a function of the nearby dipole source position. In the analysis, an integral equation for a dipole source with a metallic wall is derived and solved by applying Galerkin's MoM. A generalized network formulation is used to obtain the near field characteristics around the metallic wall due to the dipole source. Note that the induced EMF (ElectroMotive Force) method is dependent on the assumption of a sinusoidal current distribution. However, for yet larger conductors numerical solutions are required which solve for the conductor's current distribution.

In order to check the validity of the numerical calculations, the calculated near fields of the dipole source with a metallic wall are compared to experimental results.

\section{Formulation of the Problem}

In many cases, an EM source and a receptor are located near a metallic wall. Fig. 1(a) illustrates an actual situation of a receptor and an EM source near a metallic wall. Fig. 1(b) shows the geometry and coordinate system of the model used in our study for such a situation. The infinite conducting wall, which is assumed to have zero thickness, covers the entire $z=0$ plane. A dipole wire is considered, having length $L$ and radius $r$, where the axis is in the $y$ direction. It is assumed here that the wire is thin and the current flows only in the axial direction. The dipole center is located at the coordinates $x=0, y=0$, and $z=h$ in 


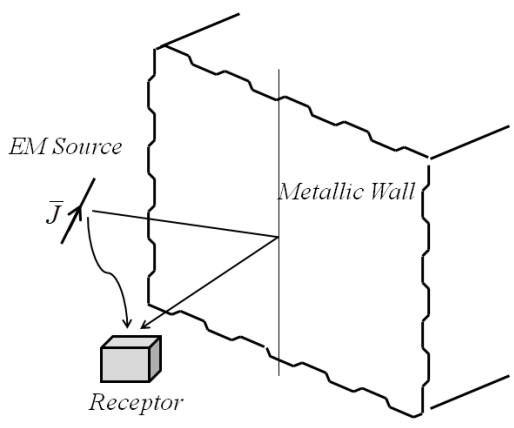

(a)

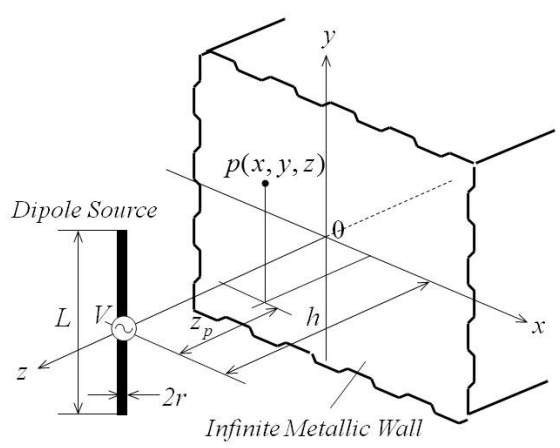

(b)

Fig. 1. (a) Actual situation of a receptor and an EM source near a metallic wall, and (b) geometry and coordinate system of the dipole source near a metallic wall.

front of the metallic conducting wall. Here $P(x, y, z)$ is the field point along the $x$-, $y$-, and $z$-axis located near the metallic wall. The dipole source and field point are placed near the metallic wall. For convenience, the auxiliary distance $z_{p}$, which denotes the position of the field point from the dipole source, is introduced. The excitation voltage $V$ is applied at the dipole center. The radiated EM field from the dipole source reflects from the metallic conducting plane. It should be noted that the structure of a parallel dipole against a metallic wall (i.e., a horizontal dipole against a metallic wall), is the worst situation for field coupling with a metallic conducting wall. Therefore, only the horizontal dipole is considered, and the vertical dipole is not addressed in this paper. The time dependence $\exp (j \omega t)$ is assumed and omitted throughout this paper.

If the dipole wire is fed by a delta gap generator as the voltage source, the integral equation for the unknown electric current $\bar{J}$ on the dipole wire above the metallic conducting wall can be written as

$$
\begin{aligned}
& \frac{1}{j \omega \varepsilon_{0}} \oiint_{S^{\prime}}\left\{\bar{K}_{w w}\left(\bar{r}, \bar{r}^{\prime}\right)-\overline{\bar{K}}_{w w^{\prime}}\left(\bar{r}, \bar{r}^{\prime}\right)\right\} \cdot \bar{J}\left(\bar{r}^{\prime}\right) d S^{\prime} \\
& =-\hat{y} V \delta(y)
\end{aligned}
$$

where the kernel is given by

$$
\overline{\bar{K}}_{w w^{\prime}}\left(\bar{r}, \bar{r}^{\prime}\right)=\left(\overline{\bar{I}} k^{2}+\nabla \nabla\right) \cdot \overline{\bar{G}}_{w w^{\prime}}\left(\bar{r}, \bar{r}^{\prime}\right)
$$

and the subscripts $w$ and $w^{\prime}$ represent a dipole wire and an image dipole, respectively. $\overline{\bar{G}}_{w w^{\prime}}$ is the dyadic Green's function of the free space. $\hat{y}$ is a unit vector in the $y$ direction, $\delta(y)$ is Dirac's delta function, $k=\omega \sqrt{\varepsilon_{0} \mu_{0}}$, and $\omega$ represents the angular frequency. The position vectors $\bar{r}$ and $\bar{r}^{\prime}$ are the observation and source points, respectively, and $d S^{\prime}$ denotes an element of an area on the dipole surface.

To solve the integral equations for the unknowns, the electric current distribution is expanded the piecewise sinusoidal functions. According to Galerkin's MoM procedure, a set of linear equations is obtained for the unknown expansion coefficients, expressed in the matrix form.

Once the current distribution on the dipole wire is given, the near electric field around the metallic wall and the dipole source can be determined. The electric field strengths are important from the EMC point of view. It is necessary to evaluate the electric fields around metallic walls due to a nearby dipole source. We are only concerned with the electric field, and the magnetic field is not considered in this paper.

When a dipole wire is excited toward a metallic wall, the electric field from the dipole source near the metallic conducting wall is obtained having the following form:

$$
\begin{aligned}
\bar{E}_{t}(\bar{r}) & =\frac{1}{j \omega \varepsilon_{0}} \oiint_{S^{\prime}}\left\{\overline{\bar{K}}_{w w}\left(\bar{r}, \bar{r}^{\prime}\right)-\overline{\bar{K}}_{w w^{\prime}}\left(\bar{r}, \bar{r}^{\prime}\right)\right\} \cdot \bar{J}\left(\bar{r}^{\prime}\right) d S^{\prime} \\
& =\bar{e}_{1}(\bar{r})-\bar{e}_{2}(\bar{r})
\end{aligned}
$$

where

$$
\begin{aligned}
\bar{e}_{1,2}(\bar{r})= & \hat{x} \frac{1}{j \omega \varepsilon_{0}} \int_{y_{n-1}}^{y_{n+1}} \frac{\partial^{2}}{\partial x \partial y}\left(\frac{e^{-j k R_{1,2}}}{4 \pi R_{1,2}}\right) F_{n}\left(y^{\prime}\right) d y^{\prime} \\
& +\hat{y} \frac{1}{j \omega \varepsilon_{0}} \int_{y_{n-1}}^{y_{n+1}}\left(\frac{\partial^{2}}{\partial y^{2}} k^{2}\right) \frac{e^{-j k R_{1,2}}}{4 \pi R_{1,2}} F_{n}\left(y^{\prime}\right) d y^{\prime} \\
& +\hat{z} \frac{1}{j \omega \varepsilon_{0}} \int_{y_{n-1}}^{y_{n+1}} \frac{\partial^{2}}{\partial z \partial y}\left(\frac{e^{-j k R_{1,2}}}{4 \pi R_{1,2}}\right) F_{n}\left(y^{\prime}\right) d y^{\prime} \\
R_{1}= & \sqrt{x^{2}+\left(y-y^{\prime}\right)^{2}+(z-h)^{2}} \\
R_{2}= & \sqrt{x^{2}+\left(y-y^{\prime}\right)^{2}+(z+h)^{2}}
\end{aligned}
$$

With the electric current, obtained from integral Eq. (1) for the unknown electric current on the dipole wire via Galerkin's MoM, the near field characteristics can be evaluated.

The maximum near fields are also important from the EMC point of view. In order to avoid a malfunction of receptors (the EUTs), it is necessary to avoid the maximum electric field location. We are only concerned with the maximum electric field. The near electric fields are normalized to their maximum field, and the concern is how near electric fields be have around the metallic conducting wall and dipole source. The normalized near field is 
defined as follows:

$$
\bar{E}_{n}(\bar{r})=\frac{\bar{E}_{t}(\bar{r})}{\bar{E}_{\max }(\bar{r})}
$$

where, $\bar{E}_{\max }$ is the maximum electric field strength, and $\bar{E}_{t}$ is a total electric field, as shown in Eq. (3).

\section{Numerical Results and Discussion}

Because wave polarization is generally difficult to predict, the worst case is usually assumed in numerical calculations. The dipole wire is located parallel to the metallic conducting wall because the coupling between the dipole and metallic wall reaches a maxima. The dipole wire used in the numerical calculation is a thin wire in comparison to the wavelength; in fact, the dimensions of the dipole wire are: $L=0.5 \lambda$ and $r=0.005 \lambda$ in the following numerical calculations.

Fig. 2 shows the computed total electric near fields $E_{t}$ of a center-fed half-wavelength dipole source located near a metallic wall as a parameter of various values of the dipole source position $h$. The maximum total near electric field occurs at the center of the dipole. As shown in Fig. 2(a), in

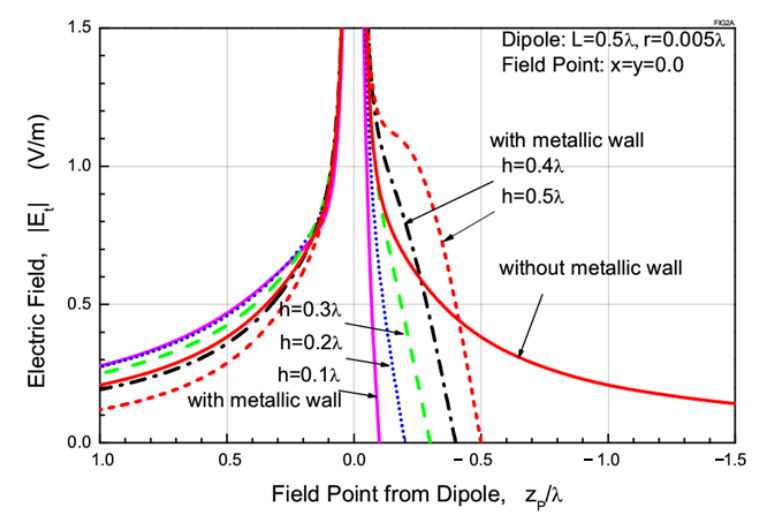

(a)

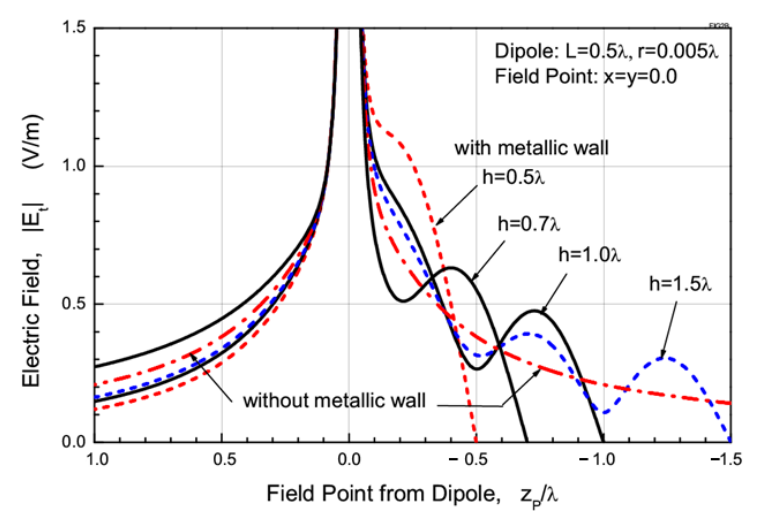

(b)

Fig. 2. Electric fields vs. field point along the $z$-axis as a parameter of various dipole source points $h$. the regions outside the dipole source (between the dipole and infinite space), total electric near field $E_{t}$ decreases gradually in magnitude with an increasing $z_{p}$ (field point from the dipole source). But in the regions inside the dipole source (between the dipole and metallic wall), total electric near field $E_{t}$ decreases rapidly with a dipole position of $h \leq 0.3 \lambda$. These falloff patterns occur in the region close to the metallic wall for closely coupled dipole-metallic wall structures $(h \leq 0.3 \lambda)$. Above $h \geq 0.4 \lambda$, total near field $E_{t}$ also decreases rapidly, but the region where $E_{t}$ is greater than that without a metallic wall exists in the region between the dipole and the metallic wall (regions inside the dipole source).

As shown in Fig. 2(b), for a dipole position of $h \geq 0.7 \lambda$, the peaks and nulls of the total near electric field $E_{t}$ occur periodically in the regions inside the dipole source (between the dipole and the metallic wall). The cause of the periodic fluctuation patterns may be due to the influence of the metallic conducting plane, especially to the mutual coupling effects between the dipole source and the metallic conducting wall. These peaks are about 1.7 times greater than without a metallic wall, and the nulls are about 0.5 times smaller than without a metallic wall. The total near electric field $E_{t}$ periodically fluctuates according to the pattern without a metallic wall, and the fluctuation period is almost $0.5 \lambda$. These periodic near field patterns due to the metallic wall smoothly and gradually approach the pattern without a metallic wall. From the EMC point of view, the peaks near the metallic wall should be considered even more carefully to prevent malfunction of the EUT receptor. Consequently, a receptor located near the metallic wall should be treated more carefully than the usual case without a metallic wall.

Fig. 3 shows the total near electric field $E_{t}$ for a zdirected field point as a parameter of various dipole positions. The peaks occur at the dipole center, and fall-off is more rapid in the regions inside the dipole source (between the dipole and the metallic wall). In the regions outside the dipole source (between the dipole and an infinite space), total near electric field $E_{t}$ decreases gradually in magnitude, with an increasing $z$ (field point

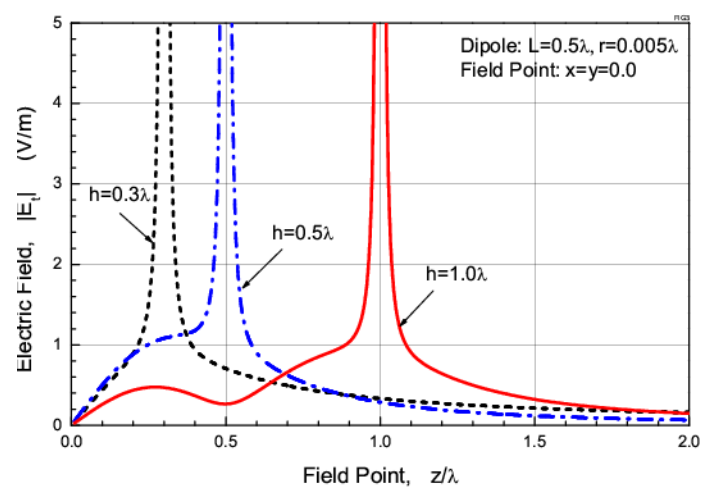

Fig. 3. Electric fields vs. field point along the $z$-axis as a parameter of various dipole positions. 
from the metallic wall). The near field patterns against the dipole source position are almost the same in the near regions of the dipole. With a dipole position of $h=1.0 \lambda$, the electric near field at $z=0.5 \lambda$ falls off due to the mutual coupling effect between the dipole source and the metallic wall. It is similar to the radiation resistance of the dipole antenna as a function of antenna height [2].

Figs. 4 and 5 show the behavior of normalized nearelectric fields (normalized to their maximum value, see Equation (6)) along two perpendicular directions: (i) along $x$-variations with $y$ parameters in the principal $H$-plane $(x z-$ plane), and (ii) along $y$-variations with $x$ parameters in the principal $E$-plane ( $y z$-plane) for $h=0.5 \lambda$ and $h=1.0 \lambda$, respectively.

Fig. 4 shows the principal $H$-plane electric field $E_{n}$ at $\mathrm{z}=0.3 \lambda$ for field points along the $x$-axis as a parameter of various field points along the $y$-axis. The normalized electric near fields for a dipole position of $h=1.0 \lambda$ at field point $x=0 \lambda$ and $y= \pm 0.5 \lambda$ are about 1.7 times greater than if $h=0.5 \lambda$, about 2.5 times greater at field point $x= \pm 1 \lambda$ and $y= \pm 0.5 \lambda$, and about 3.4 times greater at field point $x= \pm 2 \lambda$ and $y= \pm 0.5 \lambda$.

In an EMC design, the maximum near fields are of considerable interest. In order to avoid the malfunction of an EUT receptor, it is necessary to avoid the maximum

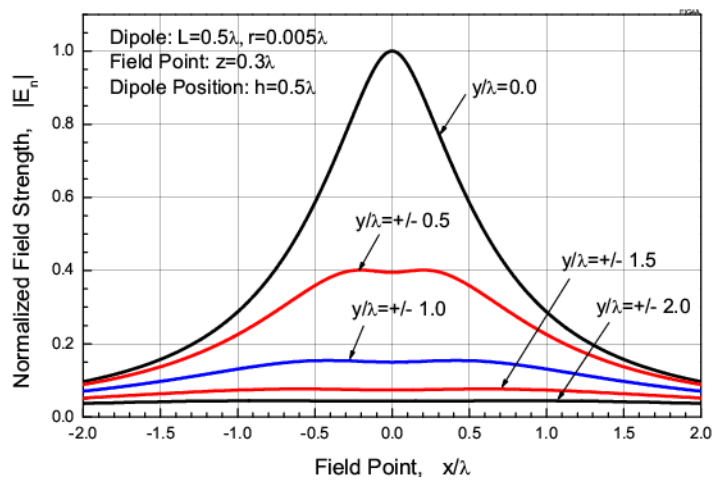

(a)

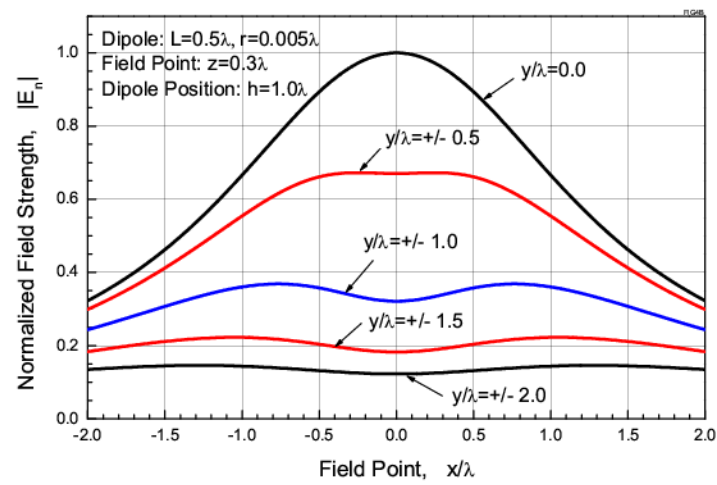

(b)

Fig. 4. Principal $H$-plane electric fields as a parameter of various field points along the $y$-axis: (a) $h=0.5 \lambda$; (b) $h=1.0 \lambda$. field location. As shown in Fig. 4, the worst position for a receptor location is along the $\mathrm{z}$-axis, and the maximum total electric field occurs at $x=y=0$. The ranges of a half magnitude of the maximum near electric field for a dipole position of $h=0.5 \lambda$ and $y=0 \lambda$ are $-0.607 \lambda \leq x \leq 0.607 \lambda$, and $-1.394 \lambda \leq x \leq 1.394 \lambda$ for $h=1.0 \lambda$ and $y=0 \lambda$, respectively. The half-magnitude range for $h=1.0 \lambda$ is 2.3 times broader than that of $h=0.5 \lambda$ in the principal $H$-plane.

Fig. 5 shows the principal $E$-plane electric field $E_{n}$ at $\mathrm{z}=0.3 \lambda$ for field points along the $y$-axis as a parameter of various field points along the $x$-axis. The normalized electric near fields for a dipole position of $h=1.0 \lambda$ at field point $y=0 \lambda$ and $x= \pm 0.5 \lambda$ are about 1.5 times greater than if $h=0.5 \lambda$, and about 2.3 times greater at field point $y= \pm 1 \lambda$ and $x= \pm 0.5 \lambda$, and about 3.0 times greater at field point $y= \pm 2 \lambda$ and $x= \pm 0.5 \lambda$.

As shown in Fig. 5, the worst position for a receptor location is also along the $\mathrm{z}$-axis, and the maximum total electric field occurs at $x=y=0$. The ranges of a half magnitude of the maximum near electric field for a dipole position of $h=0.5 \lambda$ and $x=0 \lambda$ are $-0.417 \lambda \leq x \leq 0.417 \lambda$, and $-0.698 \lambda \leq x \leq 0.698 \lambda$ for $h=1.0 \lambda$ and $x=0 \lambda$, respectively. The half-magnitude range for $h=1.0 \lambda$ is 1.7 times broader than $h=0.5 \lambda$ in the principal $E$-plane. The range of a half-magnitude of the maximum near electric

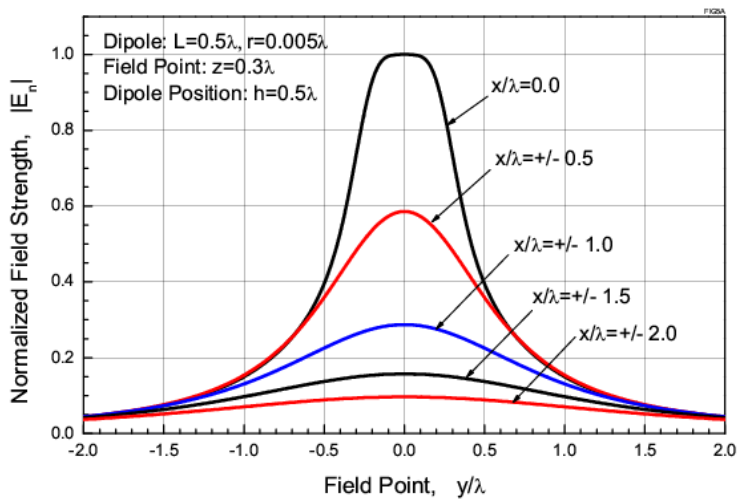

(a)

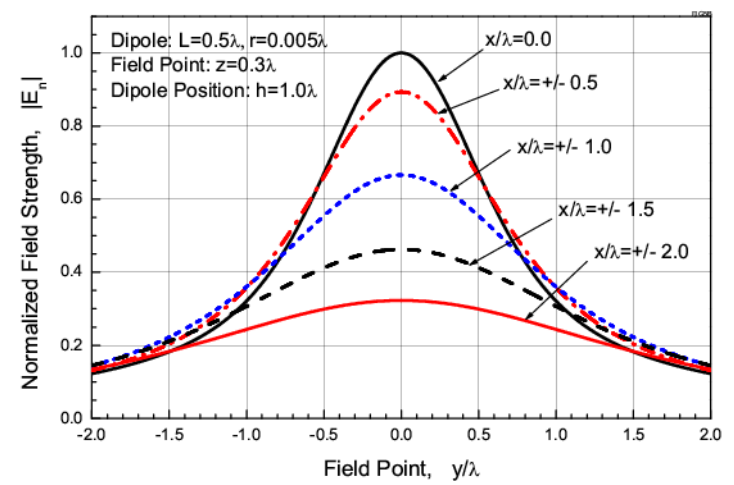

(b)

Fig. 5. Principal $E$-plane electric fields as a parameter of various field points along the $x$-axis: (a) $h=0.5 \lambda$; (b) $h=1.0 \lambda$. 


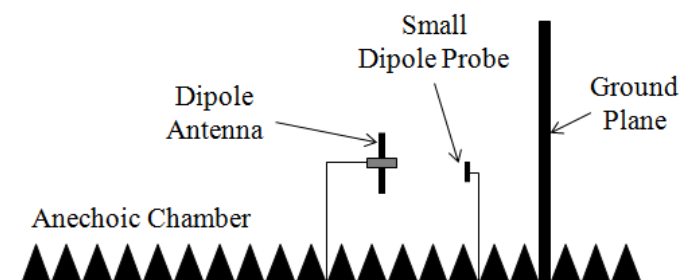

MWMWMWMWMM

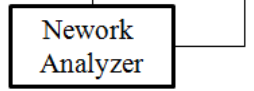

Fig. 6. Measurement setup.

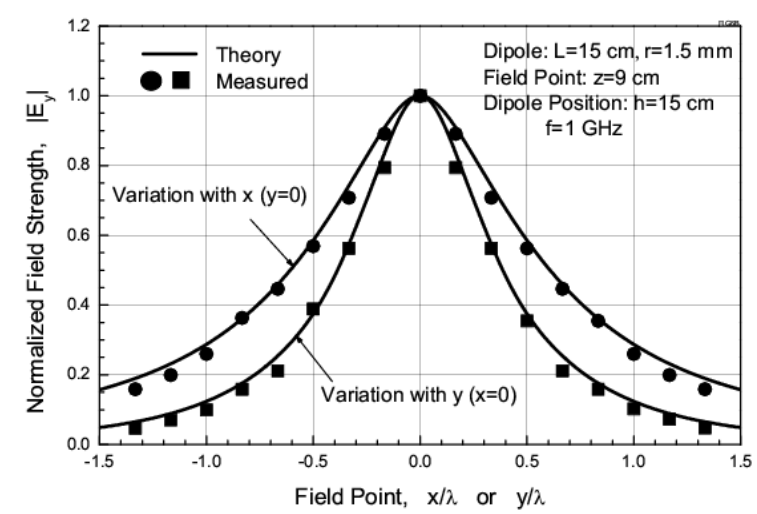

Fig. 7. Calculated and measured electric fields vs. field point along the $y$-axis.

field in the principal $H$-plane is about two times broader than that of the principal E-plane, as shown in Figs. 4 and 5.

The calculated electric field in a nearby metallic wall due to a dipole source has been compared to experimental results. The measurement setup is shown in Fig. 6 according to [13], where a half-wavelength dipole antenna at $1 \mathrm{GHz}$ was used as a dipole source, and a balanced small dipole probe (length $5 \mathrm{~mm}$ ) was used for field measurements. A large ground plane $(2 \times 4 \mathrm{~m})$ was used as a metallic wall. The half-wavelength dipole antenna was directly connected to the network analyzer (port 1). The small dipole probe was connected to the port 2, and $\mathrm{S}_{21}$ measurements along the $x$ - and $y$ - axes were recorded. We can easily convert the power obtained from $\mathrm{S}_{21}$ into electric fields. The near fields were normalized to the maximum value of the electric field at the position $x=0, y=0$, and $\mathrm{z}=9$ $\mathrm{cm}$. All measurements were made inside an anechoic chamber. For the sake of measurement, the ground plane was illuminated by a dipole source, and the near fields were measured at the center of the dipole axis using the probe. The small dipole probe was constructed by joining (via soldering) the outer ground conductors of the two semi-rigid coaxial cables. The inner conductors of both coaxial cables were extended to form a $5 \mathrm{~mm}$ balanced dipole. The other ends of the probe were connected to a network analyzer and load resistance was $50 \Omega$.

The measured and calculated electric fields are shown in
Fig. 7. For the dipole position $h=15 \mathrm{~cm}$, the near fields $E_{y}$ of a center-fed half-wave dipole are shown in Fig. 7. The results show that the calculated electric fields are in agreement with the measured data.

\section{Conclusion}

The near field characteristics around a metallic wall due to a dipole source near the metallic wall are explained. In the analysis, integral equations for a dipole source with a metallic wall are derived and solved by applying Galerkin's MoM. According to the numerical results, the near electric fields depend on both dipole position and observation point. In order to avoid malfunction of an EUT receptor, it is necessary to avoid the maximum electric field location. The worst position for a receptor location is along the z-axis, and the range for a half-magnitude of the maximum near electric field in the principal $H$-plane is about two times broader than the principal $E$-plane. These results are useful for EMC design.

\section{Acknowledgement}

This work was supported by the 2016 Yeungnam University Research Grant.

\section{References}

[1] R. F. Harrington, Time-Harmonic Electromagnetic Field, New York, Chap. 2, McGraw-Hill, 1961.

[2] E. C. Jordan and K. G. Balmain, Electromagnetic waves and radiating systems, Prentice-Hill, New York, Chap. 14, 1968.

[3] W. L. Week, Electromagnetic Theory for Engineering Applications, Chap. 7, John Wiely \& Sons, Inc., New York, 1964.

[4] C. A. Balanis, Antenna Theory, Chap. 4, John Wiley \& Sons, Inc., 2005.

[5] B. J. Strait and A. T. Adams, "Analysis and design of wire antennas with applications to EMC", IEEE Tran. Electromagn. Compat., EMC-12, no. 2, pp. 45-54, 1970.

[6] A. T. Adams, B. J. Strait, D. E. Warren, D.-C. Kuo and T. E. Baldwin, Jr, "Near fields of wire antennas by matrix methods," IEEE Tran. on Antenna and propag., AP-21, no. 5, pp. 602-610, Sep. 1973.

[7] D. E. Warren, T. E. Baldwin, and A. T. Adams, "Near electric and magnetic fields of wire antennas," IEEE Tran. on Antenna and Propag., AP-22, no. 2, p. 364, 1974.

[8] K. R. Siarkiewicz and A. T. Adams, "Near- and FarField Thin-Wire Coupling", IEEE Trans. On Electro- 
magn. Compat., EMC-19, no. 4, pp. 394-401, 1977.

[9] A. T. Adams, T. E. Baldwin, and D. E. Warren, "Near fields of thin-wire antennas-Computation and experiment," IEEE Tran. on Electro. Mag. Compa., EMC20, no. 1, pp. 259-266, Feb. 1978.

[10] A. T. Adams, Y. Leviatan, and K. S. Nordby, "Electromagnetic near fields as a function of electrical size," IEEE Tran. on Electro. Mag. Compa., EMC-25, no. 4, pp. 428-432, Nov. 1983.

[11] R. F. Harrington: "Matrix methods for field problems", Proc. IEEE, vol. 55, no. 2, pp. 136-149, 1967.

[12] R. F. Harrington: Field Computation by Moment Methods, Macmillan, New York, 1968.

[13] S. Arai, A. Tsuchiya, R. Suga, H. Sugama, and O. Hashimoto, "Experimental study on simplified evaluation method for radiation suppression ratio of NSS by using near-field measurement", IEICE Society Conference(Communications), B-4-20, p.233, 2015(in Japanese).

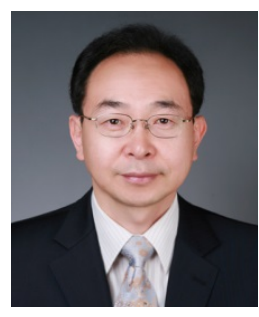

Ki-Chai Kim was born in Kyongsan, Korea. He received the B.S. degree in electronic engineering from Yeungnam University, Korea, in 1984, and M.S. and Doctor of Engineering degrees in electrical engineering from Keio University, Japan, in 1986 and 1989, respectively. He was a senior researcher at Korea Standards Research Institute, Daedok Science Town, Korea until 1993, working in electromagnetic compatibility. From 1993 to 1995, he was an Associate Professor at Fukuoka Institute of Technology, Fukuoka, Japan. Since 1995 he has been with Yeungnam University, Kyongsan, Korea, where he is currently a Professor in School of Electrical and Electronic Engineering, College of Engineering. He received the Shinohara Memorial Young Scientist Awards from the Institute of Electronics, Information and Communication Engineers (IEICE) of Japan in 1988 and received Paper Presentation Awards in 1994 from The Institute of Electrical Engineers (IEE) of Japan. He received the Achivement Awards in 1999 and 2006 from the Korea Institute of Electromagnetic Engineering and Science (KIEES). Prof. Kim was serving as the President of the Korea Institute of Electromagnetic Engineering and Science (KIEES). His research interests are in the areas of small antennas, evaluation of EMC antenna and EMI test sites, reducing electromagnetic penetration of metallic enclosure with aperture, and applications of electromagnetic field and waves.

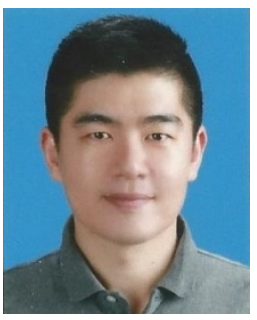

Sung Min Lim was born in Daegu, Korea. He received the B.S. and M.S. of Engineering degree in electronic engineering from Yeungnam University, Korea, in 2003, and 2005, respectively, and completed his doctorate in 2013. Mr. Lim is regular member of the Korea Institute of Electromagnetic Engineering and Science (KIEES). He was a senior engineer at SL Corporation, Kyongsan, Korea from 2014 to 2015, working in electromagnetic compatibility test team. $\mathrm{He}$ is currently a general manager at SL Corporation, working in electronics validation team. His research interests are in the EMC and validation test of automotive electronics.

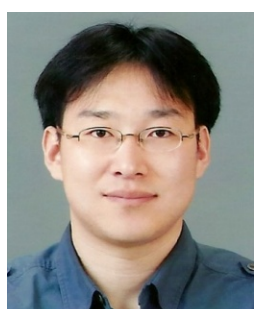

Jong-Woo Kim was born in Seoul, Korea. He received the M.S. degree in electronic engineering from Yeungnam University, Korea, in 2012, and is currently working toward a $\mathrm{Ph}$. D. He was a assistant manager at reliability center, Daesung Corporation, Ansan Korea from 2004 to 2006, working in electromagnetic compatibility. He was a team manager at KOMERI (Korea Marine Equipment Research Institute) from 2007 to 2015, working in electromagnetic compatibility test team. He is currently a center manager at KOMERI, working in marine electronics testing \& certification center. 